\title{
Características quantitativas em matrizes de codorna de corte através de análises multicaracterística
}

\author{
Quantitative traits in meat-type quail through two-trait analysis
}

\author{
Bruno Bastos Teixeira ${ }^{\text {I* }}$ Ricardo Frederico Euclydes $^{\mathrm{I}}$ Rafael Bastos Teixeira $^{\mathrm{II}}$ \\ Luciano Pinheiro da Silva ${ }^{I}$ Robledo de Almeida Torres ${ }^{I}$ Helmut Gonçalves Lehner \\ Giovani da Costa Caetano ${ }^{I}$ Aline Campores Crispim ${ }^{\text {II }}$ André Luis da Costa Paiva ${ }^{\text {II }}$
}

\section{RESUMO}

\begin{abstract}
Objetivou-se no presente trabalho avaliar o desempenho produtivo e reprodutivo de diferentes linhagens de codornas de corte, estimando os parâmetros genéticos das características mensuradas. Avaliaram-se dois grupos genéticos UFV2 e UFV1 de matrizes de codorna de corte, sendo o primeiro com 1110 animais e o segundo com 1026. Foram realizadas análises multivariadas, utilizando a metodologia de componentes principais, para definir as variáveis de maior importância. Em seguida, foram feitas análises bicaracterísticas para estimação das covariâncias e correlações genéticas, das características selecionadas. As características utilizadas na análise foram: peso da ave (P0, P7, P14, P21, P28, P35, P42, P77, P112 e P147), peso médio do ovo (POM1, POM2, POM3 e POM4), peso médio da casca (PCM1, PCM2, PCM3 e PCM4), peso médio da gema (PGM1, PGM2, PGM3 e PGM4), peso médio do albúmen (PAM1, PAM2, PAM3 e PAM4), gravidade específica média do ovo (DM1, DM2, DM3 e DM4), largura média do ovo (LOM1, LOM2, LOM3 e LOM4), comprimento médio do ovo (COM1, COM2, COM3 e COM4), número de ovos (N1, N2, N3 e N4), taxa de postura total (TXT) e idade ao primeiro ovo (IDPO). Para o grupo genético UFV1, um aumento do peso das codornas pode resultar em uma precocidade à IDPO e a seleção feita para aumento do peso corporal das codornas não resulta em perdas significativas na qualidade dos ovos. Já para UFV2, uma seleção feita aos 35 dias pode identificar as matrizes de maior peso ao longo do ciclo de produção, além de não gerar grandes perdas na taxa de postura total.
\end{abstract}

Palavras-chave: componentes principais, Coturnix coturnix, correlações genéticas.

\section{ABSTRACT}

The aim of this paper to evaluate the productive and reproductive performance of different strains of meat-type quail hens, estimating the genetic parameters measured. Two groups were evaluated genetic and UFV2 UFV1 matrix quail cutting, with 1110 being the first animal and second with 1026. Multivariate analysis were conducted using the methodology of principal components to define the most important variables. Then, two-trait analysis to estimate covariances and genetic correlations of traits. The characteristics of the analysis were: weight of the bird (P0, P7, P14, P21, P28, P35, P42, P77, P112 and P147), average egg weight (POM1, POM2, and POM3 POM4), average weight of the shell (PCM1, pcm2, and PCM3 PCM4), average weight of yolk (PGM1, PGM2, and PGM3 PGM4), weight of albumen (PAM1, PAM2, and PAM3 PAM4), average egg specific gravity (DM1,DM2,DM3 and DM4 ), average width of the egg (LOM1, LOM2, and LOM3 LOM4), average length of egg (COM1, COM2, COM3 and COM4), eggs (N1, N2, N3 and N4), total egg production (TXT) and age at first egg (IDPO). For the group of genetic UFV1, an increase in weight of birds may result in an early and that IDPO to select for increased body weight of birds does not result in significant losses in egg quality. As for UFV2, a selection made at 35 days can identify the most weight matrices throughout the production cycle, and do not generate large losses in total egg production.

Key words: main components, Coturnix coturnix, genetic correlations.

\section{INTRODUÇÃO}

As codornas são uma alternativa para produtores de aves, devido a seu crescimento rápido, maturidade sexual precoce, pequeno intervalo de gerações, alta taxa de produção de ovos, pequenas

IDepartamento de Zootecnia, Universidade Federal de Viçosa (UFV), 36571-000, Viçosa, MG, Brasil. E-mail: brunobatzootecnia@yahoo.com.br. *Autor para correspondência.

"Departamento de Zootecnia, Instituto Federal Minas Gerais, Campus Bambuí, MG, Brasil. 
exigências de espaço e nutricionais (WINTER et al., 2006). O sucesso da criação comercial de codornas de corte é dependente da disponibilidade de material genético de qualidade que garanta o potencial de produção (PAIVA et al., 2004).

OBrasil não dispõe de material genético próprio, o que deixa a coturnicultura dependente da importação de material genético. Entretanto, algumas instituições começaram a desenvolver programas de melhoramento genético de codornas de corte, visando a solucionar esse problema da dependência de material genético.

Esses programas de melhoramento necessitam de um constante acompanhamento das características de importância econômica de cada linhagem. A partir desses estudos é que os critérios de seleção são estabelecidos para garantir a renovação dos plantéis com animais de potencial genético superior geração após geração (VIANA et al., 2000).

$\mathrm{Na}$ literatura, existem poucos dados de desempenho produtivo para matrizes de corte, na fase de postura. A estimação dos parâmetros genéticos das características de postura são fundamentais para implantação do programa de melhoramento genético de codornas de corte, pois uma boa matriz de corte deve possuir uma boa postura e uma boa qualidade dos ovos produzidos (COSTA et al., 2008).

HIDALGO et al. (2007) explanaram que o desenvolvimento das linhagens implica a estimação de parâmetros genéticos e fenotípicos de forma a orientar as decisões de seleção que serão tomadas a partir das avaliações genéticas, com base nos dados coletados por meio de testes de desempenho.

Objetivou-se, no presente trabalho, avaliar os parâmetros genéticos de desempenho produtivo e reprodutivo de diferentes linhagens de codornas de corte e verificar a possibilidade de redução no número de características mensuradas no programa de melhoramento genético de codornas da Universidade Federal de Viçosa.

\section{MATERIAL E MÉTODOS}

Foram avaliados dois grupos genéticos UFV2 e UFV1 de matrizes de codorna de corte, sendo o primeiro arquivo composto por 1110 animais e o segundo por 1026. Os grupos são oriundos do programa de melhoramento genético de aves, localizados na Fazenda Boa Vista, pertencente à Universidade Federal de Viçosa. Os dados foram coletados no período de 2006 a 2009, período em que foram acompanhadas cinco gerações de codornas de corte. As aves foram pesadas nos $0^{\circ}, 7^{\circ}, 14^{\circ}, 21^{\circ}, 28^{\circ}$, $35^{\circ}, 42^{\circ}, 77^{\circ}, 112^{\circ}$ e $147^{\circ}$ dias de idade.
Foram avaliados ovos de cada codorna, coletados por três dias consecutivos, durante os quatro períodos de 35 dias, a partir do $42^{\circ}$ dia de idade. Estes foram usados para mensuração da largura e altura dos ovos e obtenção dos pesos de gema e casca.

Após a pesagem, os ovos foram cortados com estilete e suas gemas foram separadas e pesadas. As cascas identificadas foram secas ao ar e pesadas. $\mathrm{O}$ formato do ovo foi avaliado pela largura e altura com auxílio de um paquímetro digital. A gravidade específica foi obtida por meio de imersão em solução salina, com densidade variando de 1,055 a 1,085.

Foram realizadas análises multivariadas, utilizando a metodologia de componentes principais para definir as variáveis de maior importância. Em razão do grande número de variáveis medidas em unidades diferentes, foi necessária a padronização dessas variáveis $\mathrm{Xj}(\mathrm{j}=1,2, \ldots, \mathrm{p})$; nesse caso, a estrutura de dependência Xj foi dada pela matriz de correlação R.

As características utilizadas na análise foram: peso da ave (P0, P7, P14, P21, P28, P35, P42, P77, P112 e P147), peso médio do ovo (POM1, POM2, POM3 e POM4), peso médio da casca (PCM1, PCM2, PCM3 e PCM4), peso médio da gema (PGM1, PGM2, PGM3 e PGM4), peso médio do albúmen (PAM1, PAM2, PAM3 e PAM4), gravidade específica média do ovo (DM1, DM2, DM3 e DM4), largura média do ovo (LOM1, LOM2, LOM3 e LOM4), comprimento médio do ovo (COM1, COM2, COM3 e COM4), número de ovos (N1, N2, N3 e N4), taxa de postura total (TXT) e idade ao primeiro ovo (IDPO). As análises multivariadas foram feitas usando o procedimento PRINCOMP do programa SAS, versão 8.0, licenciado pela Universidade Federal de Viçosa (SAS Institute Inc., Cary, NC, USA, 1999).

Para o descarte de variáveis, primeiramente foi feito o diagnóstico de multicolinearidade (MONTGOMERY \& PECK, 1981) para evitarque variáveis linearmente dependentes possam levar a resultados inapropriados ou a conclusões que não seriam pertinentes (CRUZ \& CARNEIRO, 2006). Depois, adotou-se a recomendação de JOLLIFFE $(1972,1973)$, pela qual o número de variáveis descartadas deveria ser igual ao número de componentes cujo autovalor fosse menor que 0,7. Com esse procedimento, faz-se a eliminação das variáveis que representam menor proporção na variância total do conjunto de dados.

Logo em seguida, foram realizadas análises bicaracterísticas para estimação das covariâncias e correlações genéticas, das características selecionadas pela técnica de componentes principais. As estimativas dos componentes de (co)variância e dos parâmetros genéticos foram obtidas pelo programa MTDFREML (Multiple Trait Derivative-Free Restricted Maximum 
Likelihood), descrito por BOLDMAN et al. (1995), que utilizaram a metodologia da máxima verossimilhança restrita livre de derivadas (DFREML). O MTDFREML utiliza o algoritmo simplex para localizar o mínimo de -2 $\log _{\mathrm{e}} \mathrm{L}$ ( $\mathrm{L}=$ função de verossimilhança), e os componentes de (co)variância que minimizam a função -2 $\log _{\mathrm{e}} \mathrm{L}$ são estimativas de máxima verossimilhança.

\section{RESULTADOS E DISCUSSÃO}

Após a análise de multicolinearidade, para o grupo genético UFV1, conforme a tabela 1 , foram identificadas e eliminadas as variáveis PAM4, POM3, PAM1, POM2, POM1, LOM2, P21, P28 e LOM3 que provocavam severa multicolinearidade entre as características analisadas.

Dos 31 componentes principais, 22 (70,96\%) apresentaram autovalor menor do que 0,7 e foram descartados seguindo critério proposto por JOLLIFFE (1972 e 1973). As 22 variáveis que apresentaram maiores coeficientes, em valor absoluto, a partir do último componente principal, são passíveis de descarte. As variáveis sugeridas para descarte, em ordem de menor importância para explicar a variância total, são: COM4, TXT, POM4, PGM4, P42, PGM3, P112, PCM3, PAM2, PCM4, COM1, P35, LOM1, DM4, DM3, PCM2, DM1, DM2, PCM1, P0, PAM3 e COM3.

A análise de componentes principais indica como importantes as variáveis $\mathrm{P} 7, \mathrm{P} 14, \mathrm{P} 77, \mathrm{P} 147$, LOM4, COM2, PGM1, PGM2 e IDPO, devendo estas serem mantidas em futuros trabalhos. De um total de 31 características mensuradas, 9 foram responsáveis por $81,85 \%$ da variância total, corroborando resultados obtidos por TEIXEIRA et al. (2007), que, trabalhando com 28 características de produção de codornas de corte, encontraram 10 componentes principais que explicaram $80,78 \%$ da variação total.

RIBEIRO et al. (2008), trabalhando com 25 características de produção de codornas de corte encontraram nove componentes principais que explicaram $82,23 \%$ da variação total. Tais resultados indicam que as variáveis identificadas como as mais importantes devem ser mantidas em futuros trabalhos para discriminar indivíduos na avaliação genética.

Também, de acordo com a tabela 1, observaram-se correlações genéticas negativas entre os pesos corporais e a característica IDPO, corroborando resultados obtidos por DHALIWAL et al. (2002); MINVIELLE et al. (2002), ou seja, o aumento do peso das codornas resulta em precocidade à IDPO. Sabe-se que o peso das codornas é utilizado, até o presente, como único critério de seleção no programa de melhoramento de codornas da UFV.
Observaram-se correlações genéticas positivas e correlações genéticas negativas de baixa magnitude entre as características de qualidade de ovo e pesos corporais. PRAHARAJ et al. (1990), trabalhando com codornas japonesas, encontraram correlações genéticas positivas e negativas de baixa magnitude, entre as características de qualidade de ovo e pesos corporais. Tais resultados indicam que a seleção para aumento do peso corporal das aves não resultaria em perdas significativas na qualidade dos ovos. No entanto, o trabalho para melhoramento da qualidade dos ovos dessa linhagem deve ser feito de maneira criteriosa, para se evitarem perdas nas características de corte.

Após a análise de multicolinearidade, para o grupo genético UFV2 conforme a tabela 2, foram identificadas e eliminadas as variáveis POM4, POM2, POM3, PAM3, POM1, LOM4, P21, P28, LOM2 e P42 que provocavam severa multicolinearidade entre as características analisadas. Dos 30 componentes principais, 21 (70\%) apresentaram autovalor menor do que 0,7 e foram descartados, seguindo critério proposto por JOLLIFFE (1972 e 1973). As 21 variáveis que apresentaram maiores coeficientes, em valor absoluto, a partir do último componente principal, são passíveis de descarte.

As variáveis sugeridas para descarte, em ordem de menor importância, para explicar a variância total, são: COM4, LOM3, PGM1, PGM3, PAM1, PCM4, P112, PCM2, IDPO, LOM1, DM3, DM4, PAM2, DM2, P77, PCM1, PGM2, DM1, PGM4, P0 e P14. A análise de componentes principais indica como importantes as variáveis $\mathrm{P} 7, \mathrm{P} 35, \mathrm{P} 147$, COM1, COM2, COM3, PCM3, PAM4 e TXT. De um total de 30 características mensuradas, 9 foram responsáveis por $79,91 \%$ da variância total.

Também, de acordo com a tabela 2 as características da qualidade dos ovos apresentaram valores de correlações genéticas positivas e altas entre si, e mostraram que a seleção de apenas uma característica resultaria em ganhos correlacionados nas demais características. Esse resultado sugere que algumas mensurações, que são trabalhosas e dependentes do avaliador, não precisariam ser medidas. Essas características de qualidade dos ovos correlacionam-se genética e negativamente com a taxa de postura total. Assim, o que poderia ser obtido pela seleção dessas características provocaria uma redução na produção de ovos (TEIXEIRA, 2008).

A característica P35 correlaciona-se genética e positivamente com a característica $\mathrm{P} 147$, o que sugere que a seleção feita aos 35 dias identificaria as matrizes de maior peso ao longo do ciclo de 
Tabela 1 - Componentes principais (CP), autovalores $\left(\lambda_{2}\right)$ e percentagem da variância explicada pelos componentes (\%VCP) das características de produção em matrizes de codorna de corte do Grupo Genético UFV1.

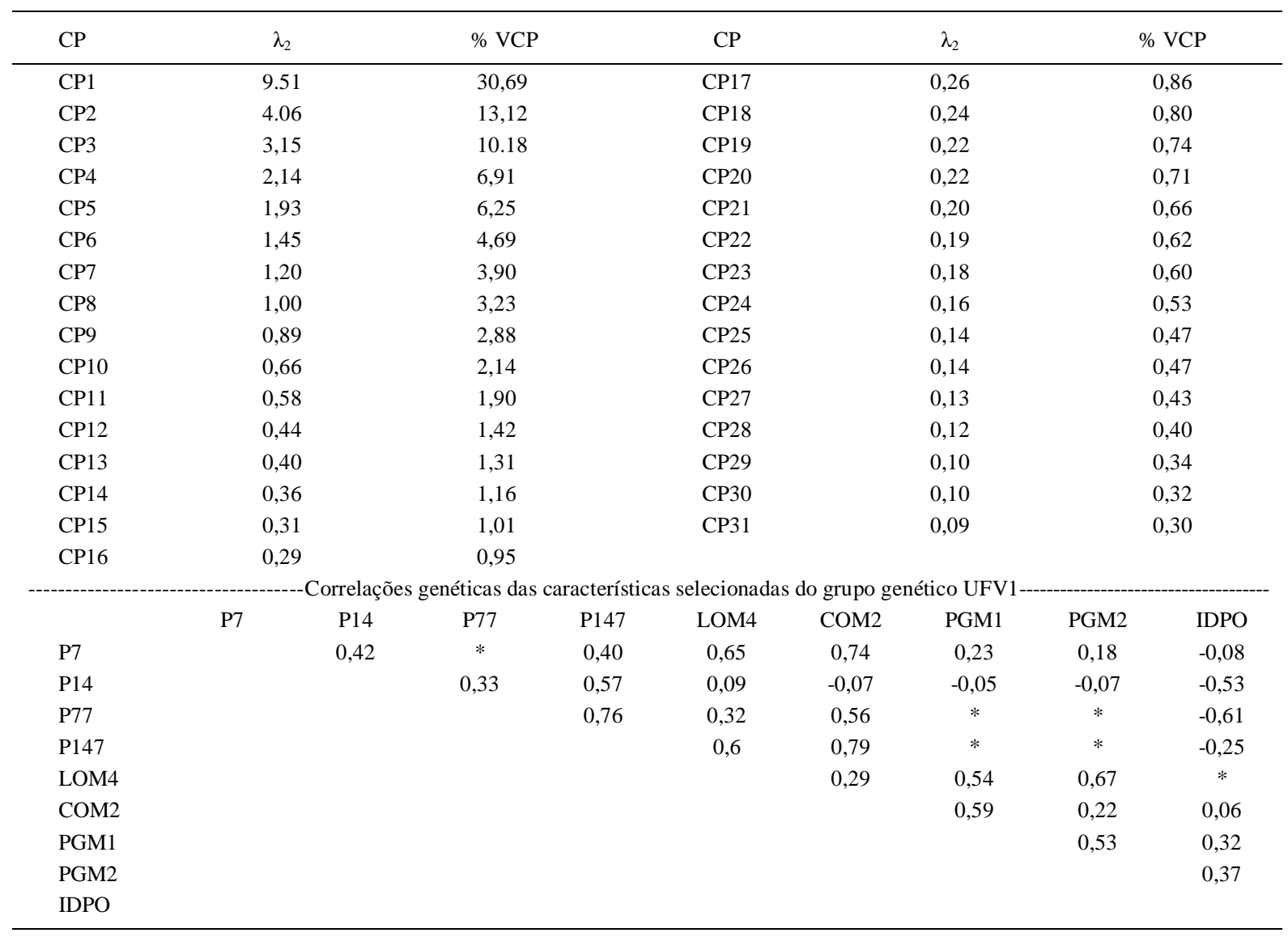

*não convergiu segundo critérios utilizados na análise bicaracterística.

produção. Segundo BONAFÉ et al. (2011a), a menor correlação entre os valores das primeiras pesagens observados foram possivelmente atribuídos à maior vulnerabilidade dos animais e ao ganho de peso diário mais intenso nos primeiros dias de vida.

BONAFÉ et al. (2011b) obtiveram valores de correlação genética positiva, de média a alta magnitude, entre peso vivo em diferentes idades de codornas de corte e valores de alta magnitude de herdabilidade dessas características, que indicam que esses animais podem ser selecionados em idades menores do que 42 dias.

Também foi observada correlação genética positiva entre P35 e TXT, o que sugere que o peso da matriz, aos 35 dias, é fator determinante para atingir a maturidade sexual e iniciar precocemente a produção de ovos, aumentando, consequentemente, a taxa de postura, sendo avaliada a maturidade sexual pela idade da postura do primeiro ovo ou quando a ave atinge $5 \%$ de postura. MINVIELLE (1998) obteve estimativas de correlação genética do número de ovos e do peso corporal, que variaram de 0 a $-0,21$, o que indica margem razoável de ganhos genéticos no número de ovos, sem grandes alterações no peso corporal, diferente do que foi observado no presente trabalho.

É importante avaliar o peso da matriz ao longo do ciclo de produção, pois, ao analisarmos a correlação genética obtida entre P147 e TXT, esta se apresenta média e negativa, gerando perdas de produção ao longo do ciclo produtivo. Entretanto, as correlações entre P7 e TXT, e P35 e TXT se apresentaram positivas e favoráveis, ou seja, a seleção para peso aos 35 dias não geraria grandes perdas na taxa de postura total. Correlações genéticas positivas entre as características de qualidade de ovos e pesos corporais indicam que a seleção para aumento do peso corporal das codornas não provocaria perdas na qualidade dos ovos.

Ciência Rural, v.42, n.12, dez, 2012. 
Tabela 2 - Componentes principais (CP), autovalores $\left(\lambda_{2}\right)$ e percentagem da variância explicada pelos componentes (\%VCP) das características de produção em matrizes de codorna de corte do Grupo Genético UFV2.

\begin{tabular}{|c|c|c|c|c|c|c|c|c|c|c|}
\hline $\mathrm{CP}$ & \multicolumn{3}{|c|}{$\lambda_{2}$} & \multicolumn{2}{|l|}{$\% \mathrm{VCP}$} & \multicolumn{2}{|l|}{$\mathrm{CP}$} & \multicolumn{2}{|l|}{$\lambda_{2}$} & $\% \mathrm{VCP}$ \\
\hline $\mathrm{CP} 1$ & \multicolumn{3}{|c|}{9.10} & \multicolumn{2}{|l|}{30,35} & \multicolumn{2}{|l|}{ CP16 } & \multicolumn{2}{|l|}{0,33} & 1,13 \\
\hline $\mathrm{CP} 2$ & \multicolumn{3}{|c|}{3.52} & \multicolumn{2}{|l|}{11,77} & \multicolumn{2}{|l|}{ CP17 } & \multicolumn{2}{|l|}{0,30} & 1,01 \\
\hline CP3 & \multicolumn{3}{|c|}{2,78} & \multicolumn{2}{|l|}{9.27} & \multicolumn{2}{|l|}{ CP18 } & \multicolumn{2}{|l|}{0,27} & 0,92 \\
\hline $\mathrm{CP} 4$ & \multicolumn{3}{|c|}{2,46} & \multicolumn{2}{|l|}{8,21} & \multicolumn{2}{|l|}{ CP19 } & \multicolumn{2}{|l|}{0,26} & 0,89 \\
\hline CP5 & \multicolumn{3}{|c|}{1,85} & \multicolumn{2}{|l|}{6,17} & \multicolumn{2}{|l|}{$\mathrm{CP} 20$} & \multicolumn{2}{|l|}{0,24} & 0,81 \\
\hline CP6 & \multicolumn{3}{|c|}{1,55} & 5,18 & & $\mathrm{CP} 21$ & & 0,21 & & 0,72 \\
\hline CP7 & & 0,97 & & 3,24 & & $\mathrm{CP} 22$ & & 0,19 & & 0,66 \\
\hline CP8 & & 0.93 & & 3,12 & & $\mathrm{CP} 23$ & & 0,18 & & 0,63 \\
\hline CP9 & & 0,78 & & 2,60 & & $\mathrm{CP} 24$ & & 0,16 & & 0,56 \\
\hline CP10 & & 0,67 & & 2,26 & & $\mathrm{CP} 25$ & & 0,15 & & 0,51 \\
\hline CP11 & & 0,63 & & 2,12 & & CP26 & & 0,14 & & 0,50 \\
\hline CP12 & & 0,50 & & 1,68 & & CP27 & & 0,12 & & 0,43 \\
\hline CP13 & & 0,45 & & 1,53 & & CP28 & & 0,12 & & 0,41 \\
\hline CP14 & & 0,43 & & 1,45 & & СР29 & & 0,10 & & 0,34 \\
\hline CP15 & & 0,36 & & 1,21 & & CP30 & & 0,09 & & 0,31 \\
\hline & P7 & & $\begin{array}{c}\text { rrelaçc } \\
\text { P35 }\end{array}$ & $\begin{array}{l}\text { éticas das c } \\
\text { P147 }\end{array}$ & $\begin{array}{l}\text { acteristic } \\
\text { COM1 }\end{array}$ & $\begin{array}{l}\text { lecionad } \\
\text { COM2 }\end{array}$ & $\begin{array}{l}\text { grupo ge } \\
\text { COM3 }\end{array}$ & $\begin{array}{l}\text { PCM3 } \\
\text { (1CO UFV }\end{array}$ & PAM4 & TXT \\
\hline P7 & & & 0,43 & 0,34 & 0,68 & 0,46 & 0,13 & 0,05 & 0,14 & 0,28 \\
\hline P35 & & & & 0,90 & $*$ & 0,38 & 0,25 & 0,09 & 0,5 & 0,26 \\
\hline P147 & & & & & $*$ & $*$ & $*$ & $*$ & $*$ & $-0,53$ \\
\hline COM1 & & & & & & $*$ & $*$ & 0,74 & 0,64 & $-0,17$ \\
\hline $\mathrm{COM} 2$ & & & & & & & 0,81 & 0,53 & 0,30 & $-0,27$ \\
\hline COM3 & & & & & & & & 0,59 & 0,22 & $-0,81$ \\
\hline PCM3 & & & & & & & & & 0,34 & $-0,22$ \\
\hline PAM4 & & & & & & & & & & $-0,51$ \\
\hline TXT & & & & & & & & & & \\
\hline
\end{tabular}

*não convergiu segundo critérios utilizados na análise bicaracterística.

Quando se trata de seleção de mais de uma característica, existem vários métodos para se efetuar a seleção, como os métodos de Tanden, níveis independentes de eliminação e os índices de seleção. Os dois primeiros apresentam desvantagens no caso de correlações antagônicas entre as características, nem sempre resultando em máximo ganho. No caso dos índices de seleção, o problema está no estabelecimento de pesos econômicos para as características, além da redução do ganho genético por característica, quando se inclui um número maior de características no índice.

Técnicas de análise multivariada, como a de componentes principais, têm sido sugeridas em substituição aos índices de seleção, pois permitem combinar informações múltiplas contidas nas unidades experimentais, levando os indivíduos mais promissores e mais adequados aos propósitos de melhoramento (MARTINS et al., 2003). Os resultados do presente estudo demonstram a possibilidade de redução no número de características, o que torna a seleção mais eficiente.

\section{CONCLUSÃO}

Existe a possibilidade de redução do número de características mensuradas no programa de melhoramento genético de codorna de corte. As características selecionadas pelo critério de componentes principais têm herdabilidades de moderadas a alta e a maior parte das correlações genéticas entre estas foram favoráveis aos objetivos de seleção.

\section{REFERÊNCIAS}

BOLDMAN, K.G.A. et al. A manual for use of MTDFREML. A set of programs to obtain estimates of variance and covariances. Washington, DC: ARS, USDA, 1995. 120p.

BONAFÉ, C.M. et al. Modelos de regressão aleatória para descrição da curva de crescimento de codornas de corte. Revista brasileira de zootecnia, v.40, n.4, p.765-771, 2011a. Disponível em: <http://dx.doi.org/10.1590/S1516-35982011000400009>. Acesso em: 26 jun. 2011. doi: 10.1590/ S1516-35982011000400009. 
BONAFÉ, C.M. et al. Heterogeneidade de variância residual em Modelos de Regressão Aleatória na descrição do crescimento de codornas de corte. Revista Brasileira de Zootecnia, v.40, n.10, p.2129-2134, 2011b. Disponível em: <http:// dx.doi.org/10.1590/S1516-35982011001000009>. Acesso em: 10 dez. 2011. doi: 10.1590/S1516-35982011001000009.

COSTA, C.H. et al. Avaliação do desempenho e da qualidade dos ovos de codornas de corte de dois grupos genéticos. Revista Brasileira de Zootecnia, v.37, n.10, p.1823-1828, 2008. Disponível em: <http://dx.doi.org/10.1590/S1516-35982008001000016>. Acesso em: 22 jun. 2011. doi: 10.1590/S1516-35982008001000016.

CRUZ, C.D.; CARNEIRO, P.C.S. Modelos biométricos aplicados ao melhoramento genético. 2.ed. Viçosa: UFV, 2006. $2 \mathrm{v}$.

DHALIWAL, S.K. et al. Inheritance of body weight and egg production characters and their interrelationships in Japanese quails. Journal of Livestock and Poultry Production, v.18, n.3-4, p.1-8, 2002.

HIDALGO, A.M. et al. Parâmetros genéticos para peso do ovo e peso corporal em codornas de postura. In: SIMPÓSIO INTERNACIONAL, 3.; CONGRESSO BRASILEIRO DE COTURNICULTURA, 2., 2007, Lavras, MG. Anais... Lavras: NECTA, 2007. V.3, p.219.

RIBEIRO, J.C. et al. Analise Multivariada de características de produção em codornas de corte. 2008. In: SIMPÓSIO INTERNACIONAL4.; CONGRESSO BRASILEIRO DE COTURNICULTURA, 3., 2007, Lavras, MG. Anais... Lavras: NECTA, 2007. V.4, p.250.

JOLLIFFE, I.T. Discarding variables in a principal component analysis. I: artificial data. Applied Statistics, v.21, p.160$173,1972$.

JOLLIFFE, I.T. Discarding variables in a principal component analysis. II: real data. Applied Statistics, v.22, p.21-31, 1973.

MARTINS, I.S. et al. Avaliação de critérios multivariados aplicados na seleção em Eucalyptus grandis. Hill ex Maiden. Floresta e ambiente, v.10, n.1, p.38-47, 2003.

MINVIELLE, F. Genetics and breeding of Japanese quail for production around the world. In: ASIAN PACIFIC POULTRY
CONGRESS, 6., 1998, Nagoia. Proceedings... Nagoia: Japan Poultry Science Association, 1998. p.122-127.

MINVIELLE, F. et al. Fearfulness and performance related traits in selected lines of Japanese quail (Coturnix japonica). Poultry Science, v.81, n.3, p.321-326, 2002.

MONTGOMERY, D.C.; PECK, E.A. Introduction to linear regression analysis. New York: J. Wiley, 1981. 504p.

PAIVA, E.D. et al. Estimativas de parâmetros genéticos para característica de produção e qualidade de ovo, em codornas de postura. Revista Brasileira de Ciência Avícola, v.6, n.4, p.138-138, 2004.

PRAHARAJ, N.K. et al. Developments in processing quail meat an eggs. World's Poultry Science Journal, v.46, n.11, p.219-234, 1990.

SAS. Software: versão 8.0. Cary, NC, 1999. 680p.

TEIXEIRA, R.B. Utilização da metodologia de componentes principais em características de produção da linhagem de codorna de corte UFV-1. In: SIMPÓSIO INTERNACIONAL DE COTURNICULTURA, 3., 2007, Lavras, MG. Anais... Lavras: NECTA, 2007. V.3, p.210.

TEIXEIRA, R.B. Avaliação e estimação de componentes genéticos de características produtivas e da qualidade de ovos de linhagens de codorna de corte. 2008. 58f. Dissertação (Mestrado em Zootecnia) - Curso de Pós-graduação em Zootecnia, Universidade Federal de Viçosa, MG.

VIANA, C.F.A. et al. Influência de grupos genéticos e de níveis de energia sobre características de carcaça de frangos de corte. Revista Brasileira de Zootecnia, v.29, n.4, p.1067-073, 2000. Disponível em: <http://dx.doi.org/10.1590/S151635982000000400017>. Acesso em: 19 jan. 2011. doi: 10.1590/ S1516-35982000000400017.

WINTER, E.M.W. et al. Aplicação do método Bayesiano na estimação de correlações genéticas e fenotípicas de peso em codornas de corte em várias idades. Revista da Sociedade Brasileira de Zootecnia, v.35, n.4, p.1684-1690, 2006. Disponível em: <http:/ /dx.doi.org/10.1590/S1516-35982006000600015>. Acesso em: 18 jan. 2011. doi: 10.1590/S1516-35982006000600015. 